\title{
Establishment of a Predictive Model for Factors \\ Related to Systemic Inflammatory Response \\ Syndrome (SIRS) after Percutaneous \\ Nephrolithotomy (PCNL)
}

Min Ju

The First Hospital of China Medical University

Jin Zheng

The First Hospital of China Medical University https://orcid.org/0000-0002-5643-1526

Lin Lin Gao

The First Hospital Of China Medical University

Li Ying Pan ( $\sim$ panyingli@sina.com )

The First Hospital of China Medical University

\section{Research article}

Keywords: renal stone, nephrolithotomy, percutaneous, systemic inflammatory response syndrome, sepsis

Posted Date: September 28th, 2020

DOI: https://doi.org/10.21203/rs.3.rs-29106/v3

License: (c) (1) This work is licensed under a Creative Commons Attribution 4.0 International License.

Read Full License 


\section{Abstract}

Background: Percutaneous nephrolithotomy (PCNL) is a primary treatment method for renal stones, but infection is a very common postoperative complication. Systemic inflammatory response syndrome (SIRS) is a stage of the infection process and a very important early clinical manifestation of sepsis, so identifying the risk factors associated with SIRS after PCNL is important for ensuring patient safety and preventing sepsis.

Objective: To analyze the risk factors for SIRS after PCNL, identify the predictive factors, and perform risk factor analysis.

Methodology: Between September 2016 and September 2017, 352 patients who were diagnosed with renal stones and treated with PCNL were included in this study. The patients were divided into two groups according to whether SIRS occurred. Univariate analysis was performed on the related risk factors, including patient age; gender; body mass index; urine culture; number, types and quantity of rental stones; diabetes; blood glucose; complications; hospital stay; residual stones; and Guy's degree. Then, logistic regression was used to perform multivariate analysis and establish a predictive model.

Results: A total of 352 patients with renal stones were treated with PCNL, and 106 patients (30.1\%) developed SIRS after surgery. Operative time, preoperative fever and diabetes were found to be risk factors, and the logistic regression results indicated that diabetes $(\mathrm{OR}=2.049,95 \% \mathrm{Cl} 1.008 \sim 4.166)$ and operative time $(\mathrm{OR}=1.011,95 \% \mathrm{Cl} 1.003 \sim 1.019)$ could be entered into the regression equation. Therefore,

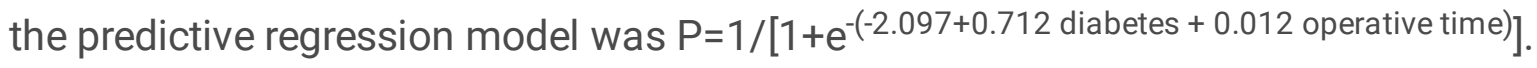

Conclusion: Diabetes and operative time are independent risk factors for SIRS after PCNL, so the probability of SIRS after PCNL can be determined according to these two indicators.

\section{Introduction}

Renal stones are one of the most common urological conditions, and they have a high incidence around the world. A cross-sectional survey of the prevalence of urinary calculi in China showed that the prevalence rate was $6.50 \%$ (1). Percutaneous nephrolithotomy (PCNL) has been widely used for the treatment of renal stones (2), and it has also become the standard of care for large $(>2 \mathrm{~cm})$ renal stones (3). PCNL has become one of the main methods used to treat renal and upper ureteral calculi in recent years. Compared to other approaches, it has the advantages of less trauma, fewer complications and faster recovery, but postoperative infection and even sepsis are problems that cannot be ignored.

Systemic inflammatory response syndrome (SIRS) refers to the systemic nonspecific inflammatory response that is caused by the stimulation of infectious and noninfectious factors, such as various severe infections, trauma, hemorrhagic shock, burns, tissue ischemia and hypoxia and reperfusion injury, which eventually leads to an uncontrolled response to inflammation. Infection is a continuous clinical process; SIRS is one of the stages, and it is also a very important early clinical manifestation of sepsis. 
The broken stones in percutaneous nephrolithotomy undergo reverse flow into the blood under the action of trauma and hyperbaric perfusion, which is likely to cause SIRS. After PCNL, $10 \%$ to $35 \%$ of patients develop SIRS, with a small percentage progressing to sepsis (4). Therefore, controlling SIRS is very important to prevent the occurrence of sepsis after PCNL and ensure the perioperative safety of patients.

At present, there are many papers discussing the risk factors for systemic inflammatory response syndrome after percutaneous nephrolithotomy, and factors such as stone size, operative time, number of channels, positive results of urine culture, renal pelvic urine, and stone cultures, are risk factors for SIRS after percutaneous nephrolithotomy $(5,6)$. It is necessary to establish a nomogram-based predictive model to predict the postoperative risk of severe infection. Prediction of the risk of SIRS after PCNL via scientific methods will help clinical staff to assess the SIRS risk of patients early during the perioperative period and implement early interventions, which can ensure patient safety and promote early rehabilitation $(5,7,8)$.

\section{Patients And Methods}

\section{Study design and study setting}

This retrospective study was approved by the hospital ethics committee (2018-237-2, No. 1 Hospital of China Medical University), and the case data of qualified patients were surveyed in the medical record room. Between September 2016 and September 2017, there were 352 cases in the Urology Department of No. 1 Hospital of China Medical University. A total of 352 cases were included for statistical analysis. The following inclusion criteria were used for patients: $\otimes$ The patients were diagnosed with renal stones based

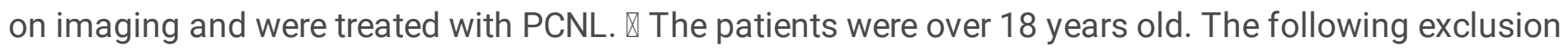
criteria were used for patients: Patients who underwent open surgery for renal calculi complicated with sepsis and American Society of Anesthesiologists (ASA) scores of 3 and 4 were excluded. All surgical patients were evaluated with routine blood examination, hemoglobin, routine urine analysis and urine culture, blood glucose, kidney function tests, stone clearance rate, Guy's degree, intravenous pyelogram and contrast-enhanced computed tomography if necessary for diagnosis.

All operations were performed by doctors who had experience with PCNL surgeries with the above characteristics as associate professors. Patients with positive urine culture before surgery were treated with appropriate antibiotics until routine urine analysis was normal and urine culture was negative. The operative method was to place the patient in the lithotomy position after induction of general anesthesia. The 5F-7F ureteral catheter was inserted in a retrograde manner under direct cystoscopy, and an isotonic solution was injected to form "artificial hydronephrosis" to indwell the catheter. With the patient in the prone position, a pillow was placed under the abdomen to form a low arc in the back at the waist. Under the guidance of B-ultrasound, puncture was performed from the posterior axillary line under rib 12 or between ribs 11 and 12 to the dorsal calyces of the affected kidney, and the number of puncture points and channels were determined according to the position, size and number of renal stones. After successful puncture, the metal guide wire was placed. After withdrawing the needle sheath, the channel 
was expanded to $24 \mathrm{~F}$, and the expansion sheath was indwelled. The position of the stone was checked. The stone was removed directly if possible, and larger stones were first crushed with a Swiss LithoClastMaster (EMS, Nyon, Switzerland) and then sucked out or pinched out. Finally, the renal pelvis was checked for residual stones, and the postoperative $14 \mathrm{~F}-16 \mathrm{~F}$ renal fistula and $5 \mathrm{~F}-7 \mathrm{~F}$ double $\mathrm{J}$ tube were indwelled.

All patients were followed up postoperatively for SIRS, which was diagnosed in patients according to the following criteria: $\otimes$ fever, $>38^{\circ} \mathrm{C}$ or $\left\langle 36^{\circ} \mathrm{C}\right.$; $\otimes$ heart rate, $>90$ beats $/ \mathrm{min}$; $\otimes$ respiratory rate, $>20$ breaths $/ \mathrm{min}$; or $\otimes$ leukocyte count, $<4 \times 10^{9} / \mathrm{L}$ or $>12 \times 10^{9} / \mathrm{L}$, or immature granulocytes $>10 \%$. SIRS was diagnosed in patients who met two or more criteria (9).

\section{Statistical analysis}

Statistical analyses were performed using the Statistical Package for SPSS 20.0 (Chinese/BM, China). Statistical significance was set at a $p$ value $<0.05$, and all $p$ values were single tailed. Measurement data were presented as $\pm S D$, and count data were expressed as the number of cases (percentage). Continuous variables were compared with an independent-sample t test, count data were subjected to univariate analysis with the chi-square test, and the rank sum test was used for rank data. To define potential risk factors for SIRS, we performed multivariate logistic regression analysis and established a predictive model. The beta ( $($ ) regression coefficient and the odds ratios (ORs) and their $95 \%$ confidence intervals (Cls) were calculated. SPSS 20.0 and Empower Stats (http://www.empowerstats.com/cn/) were used for further analysis. Missing data were managed with 5 replicates and a chained equation approach method in SPSS (10). A nomogram was formulated to provide visualized probability prediction (11). We assessed the predictive performance of the models by discrimination and calibration using the C-statistic and Hosmer-Lemeshow chi-square statistic. Discrimination was conducted to calculate the area under the AUC curve by ROC analysis. An AUC of 0.5 indicates no discrimination, whereas an AUC of 1.0 shows perfect discrimination (12). An internal validation study was conducted using 1000 bootstrap resamples to determine the final variables predictive of SIRS. Calibration was performed by a calibration plot, which assessed whether the predicted and observed probabilities were in concordance (13).

\section{Results}

(1) General information for the patients (see Table 1 for details)

Table 1 General information of the patients and univariate analysis ursults for SIRS after PCNL 


\begin{tabular}{|c|c|c|c|c|}
\hline Variable & $\operatorname{SIRS}(+)$ & SIRS(-) & total & P-value \\
\hline
\end{tabular}

Gender

$\begin{array}{lllll}\text { male } & 60 \square 56.60 \% \square & 150 \square 61.00 \% \square & 210 \square 59.7 \% \square & 0.443 \\ \text { female } & 46 \square 43.40 \% \square & 96 \square 39.00 \% \square & 142 \square 40.3 \% \square & \end{array}$

Age

$\begin{array}{ccccc}\text { Male } & 53.53 \pm 11.40 & 53.99 \pm 9.37 & 55.20 \pm 10.69 & 0.983 \\ \text { Female } & 54.63 \pm 11.91 & 55.48 \pm 10.10 & 54.4 \pm 10.27 & 0.659 \\ \text { average } & 54.01 \pm 11.58 & 54.57 \pm 9.67 & 53.86 \pm 9.97 & 0.661 \\ \text { Body Mass Index } & 24.95 \pm 4.16 & 25.35 \pm 4.98 & 25.22 \pm 4.73 & 0.477 \\ \text { Urine culture } & & & & \\ \text { positive } & 1 \square 0.90 \% \square & 8 \square 3.25 \% \square & 9 \square 2.6 \% \square & 0.373 \\ \text { negative } & 105 \square 99.10 \% \square & 238 \square 96.75 \% \square & 343 \square 97.4 \% \square & \end{array}$

Rental stones

$\begin{array}{lllll}\text { single } & 54 \square 50.94 \% \square & 129 \square 52.44 \% \square & 183 \square 52.0 \% \square & 0.797 \\ \text { multiple } & 52 \square 49.06 \% \square & 117 \square 47.56 \% \square & 169 \square 48.0 \% \square & \end{array}$

Hemoglobin

$128.61 \pm 20.34$

$19.40 \pm 18.36$

$129.16 \pm 18.97$

0.722

Blood pressure

$\begin{array}{lllll}\text { Systolic pressure } & 140.22 \pm 19.41 & 140.45 \pm 19.34 & 140.38 \pm 19.36 & 0.155 \\ \text { Diastolic pressure } & 84.03 \pm 10.00 & 83.26 \pm 11.54 & 83.49 \pm 11.08 & 0.552\end{array}$

Diabetes

$\begin{array}{lllll}\text { Yes } & 11 \square 10.38 \% \square & 48 \square 1.68 \% \square & 59 \square 16.8 \% \square & 0.035 * \\ \text { no } & 95 \square 89.62 \square & 198 \square 98.32 \% \square & 293 \square 83.2 \% \square & \end{array}$


Blood glucose

$5.83 \pm 1.62$

$5.85 \pm 1.71$

$5.84 \pm 1.68$

0.927

Hydronephrosis

\begin{tabular}{|c|c|c|c|}
\hline Yes & 78ロ73.58\%॰ & $172 \square 69.92 \% \square$ & $270 \square 76.7 \% \square$ \\
\hline no & $28 \square 26.42 \% \square$ & $74 \square 30.08 \% \square$ & 82₫23.3\%匹 \\
\hline
\end{tabular}

Renal failure

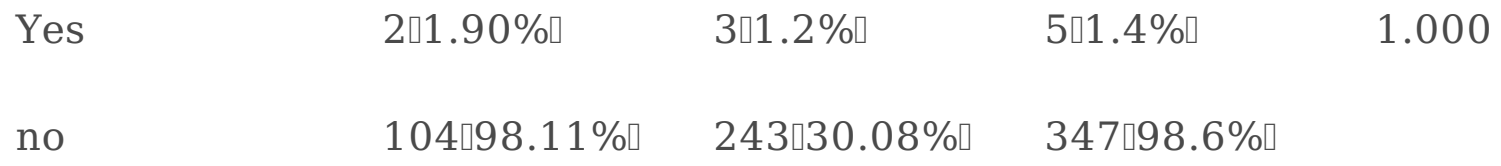

Fever before operation

\begin{tabular}{|c|c|c|c|c|}
\hline Yes & $9 \square 8.49 \% \square$ & $15 \square 6.10 \% \square$ & $24 \square 6.8 \% \square$ & $0.002^{*}$ \\
\hline no & 97๑91.51\%॰ & $231 \square 93.90 \% \square$ & 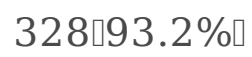 & \\
\hline
\end{tabular}

Operative time

$61.87 \pm 34.65$

$51.84 \pm 24.78$

$54.86 \pm 28.45$

$0.008 *$

Cast stone or staghorn stone

\begin{tabular}{|c|c|c|c|}
\hline Yes & $11 \square 10.38 \% \square$ & $14 \square 5.69 \% \square$ & $25 \square 7.1 \% \square$ \\
\hline no & 95ロ89.62\%ロ & 232๘94.31\%॰ & 327๑92.9\%॰ \\
\hline
\end{tabular}

History of stone surgery

\begin{tabular}{|c|c|c|c|}
\hline Yes & $45 \square 42.45 \% \square$ & $115 \square 46.75 \% \square$ & $160 \square 45.5 \% \square$ \\
\hline no & 61घ57.55\%॰ & 131053.25\%॰ & $192 \square 54.5 \% \square$ \\
\hline
\end{tabular}

Stone clearance rate

$\begin{array}{ccccc}\text { Stone removal } & 105 \square 99.06 \% \square & 241 \square 97.97 \% \square & 346 \square 98.30 \% \square & 0.783 \\ \text { residual stone } & 1 \square 0.94 \% \square & 5 \square 2.03 \% \square & 6 \square 1.70 \% \square & \\ \text { hospital stays } & 14 \square 13 \square 18 \square & 14 \square 12 \square 16 \square & \\ \text { Guy’s degree } & & & \\ 1 & 4 \square 3.77 \% \square & 6 \square 2.44 \% \square & 10 \square 2.84 \% \square & 0.019 *\end{array}$


A total of 352 patients who underwent PCNL for renal stones were included in the present study. Among them, 210 were males $(59.7 \%)$, and 142 were females (40.3\%). The average age was $53.86 \pm 9.97$ years, the average body mass index was $25.22 \pm 4.73$, and the average operative time was $54.86 \pm 28.45$. Nine patients $(2.6 \%)$ were positive for preoperative urine culture, 169 patients $(48 \%)$ had multiple renal stones, 25 patients $(7.1 \%)$ had cast stones or staghorn stones, and 59 patients $(16.8 \%)$ had concurrent diabetes. The average preoperative blood glucose level was $5.84 \pm 1.68 \mathrm{mml} / \mathrm{L}$. There were 270 cases $(76.7 \%)$ with concurrent hydronephrosis, 5 cases (1.4\%) with concurrent renal insufficiency, 24 cases (6.8\%) with preoperative fever, 160 cases (45.5\%) with a preoperative history, 106 cases $(30.1 \%)$ with SIRS, and no patients with sepsis. There was no difference between the SIRS and non-SIRS groups in terms of age, gender, or BMI.

(2) Results of univariate analysis for SIRS after percutaneous nephrolithotomy: It was found in the univariate analysis that the differences in operative time, preoperative fever, diabetes and previous stone operative history were statistically significant, with $p<0.05$, as shown in Table 2.

Table 2 Multivariate logistic regression analysis results for SIRS after PCNL

\begin{tabular}{lccc}
\hline Variable & OR & $95 \%$ confidence interval & P-value \\
\hline Diabetes & 2.038 & $1.006 \square 4.130$ & 0.048 \\
Operative time & 1.012 & $1.004 \square 1.020$ & 0.004 \\
\hline
\end{tabular}

(3) Results of multivariate logistic regression analysis for SIRS after PCNL: Variables with $p<0.2$ in the univariate analysis, including gender, systolic pressure, diabetes, fever before operation, operative time, and history of stone surgery, were selected for logistic regression. It was found in the multivariate analysis that diabetes and operative time were risk factors, as shown in Table 2; as a result, the predictive regression model $\mathrm{P}=1 /\left[1+\mathrm{e}^{-(-2.097+0.712 \text { diabetes }+0.012 \text { operative time })}\right]$ was established. For example, nondiabetic patients, 60 minutes of operation, $p=0.21$; nondiabetic patients, 120 minutes of operation, $p=0.34$; diabetic patients, 60 minutes of operation, $p=0.35$; and diabetic patients, 120 minutes of operation, $p=0.51$. The risk probability can be obtained according to the risk factors, so medical staff can perform targeted observation for SIRS in postoperative patients. 
(4) A nomogram was established incorporating two predictive factors (diabetes and operative time) (Fig 1). Operative time made the largest contribution to SIRS risk, followed by diabetes. In the plot, each clinicopathological factor corresponds to a specific point when a line is drawn straight upward to the point axis. After the sum of the points is located on the total points axis, the sum represents the probability of SIRS when survival is visualized by drawing a line straight down to the SIRS axis. For example, PCNL lasted for sixty minutes (28 points) in diabetic (28 points) patients. The point total was 56 , and the risk of SIRS was approximately $35 \%$. This predictive value could be used to make decisions for clinical treatment and patient counseling.

The C-index for the predictive model was 0.608 [95\% confidence interval (Cl): 0.504-0.748], which showed discrimination ability. The ROC curve is presented in Fig 2 . The H-L chi-square statistic was 12.834 , which indicated good statistical performance upon internal validation between the nomogram prediction and the actual observation for the probability of SIRS $(P=0.196)$. The calibration plot is shown in Fig 3 .

The red solid line is a smoothed curve that represents an estimate of the relation between the predicted and observed probability of SIRS. Ideally, this line fits the black solid line that represents perfect calibration.

\section{Discussion}

In this study, there were slightly more males than females, but there was no significant sex difference between the SIRS and non-SIRS groups. A total of $30.1 \%$ of patients developed SIRS, and no patients developed sepsis. Current studies have shown that the incidence of SIRS after PCNL is between $10 \%$ and $30 \%(6,14)$. This value was similar to the results in the literature $(15,16)$ but higher than those in the studies of Wang A]QL $(12.5 \%)$ and Singh P $(17.5 \%)(3,9)$.

Patients with a preoperative stone operation history accounted for $45.5 \%$ of the patients, indicating that the recurrence rate of renal stones was high and the treatment was complicated. There were 9 patients with positive urine cultures and 24 patients with preoperative fever and concurrent infection. Although preoperative fever was a risk factor in univariate analysis, it was not entered into the regression equation after multivariate analysis. The following factors could explain this finding: first, patients with preoperative fever were required to undergo anti-infective treatment; second, patients who were found to have bacterial infection in routine urine analysis and positive urine culture were also treated effectively; third, the patients did not undergo surgery until their test results were normal. Thus, the preoperative fever failed to become a risk factor, and no patients suffered sepsis.

The results of this study revealed two risk factors that were ultimately entered into the equation for predicting SIRS after PCNL: diabetes and operative time. Diabetes has been reported to a risk factor for SIRS after PCNL $(17,18)$. Zhu Zhenjie (18) reported that the incidence of SIRS after PCNL in patients with blood glucose above $7.1 \mathrm{mmol} / \mathrm{L}$ was more than twice that of patients with normal preoperative blood glucose, but the blood glucose value of patients with concurrent preoperative diabetes was controlled in the normal range, indicating that diabetes itself carries a certain risk of SIRS after PCNL; thus, even if the 
preoperative blood glucose is controlled in the normal range, it should still be taken seriously as a postoperative risk factor. The OR results were similar to those of Zhu Zhenjie (17).

Operative time was found to be a very important risk factor, consistent with other studies (8). The prolongation of operative time, with an increase in the absorption of lavage fluid and toxins into the blood, increases the chance of infection. Absorption of a large amount of lavage fluid can cause hypothermia and coagulation dysfunction and even increase the circulatory load and disturb the balance of water and electrolytes. SIRS is more likely to occur after surgery. Shortening the operative time is an important strategy to prevent SIRS. With the continuous improvement of endoscopy technology, clinicians are currently decreasing their operative time. However, in Chen L's research, operative time was not entered into the regression equation (6).

A total of $7.1 \%$ of the patients suffered cast stones or staghorn stones. These stones were difficult to handle and prone to cause complications, so extensive clinical experience was required for surgeons. Although their operative time was relatively long, these stones were ultimately not entered into the regression equation.

With the careful monitoring of patients for SIRS after percutaneous nephrolithotomy for renal stones, the continuous enhancement of operative skills and the improvement of preoperative preparation, although nearly one-third of patients developed SIRS after percutaneous nephrolithotomy, the number of patients with sepsis was zero, indicating that PCNL surgery is becoming a more mature and safer procedure. Early symptomatic treatment for SIRS can prevent the patient's condition from deteriorating further, so it is necessary to use predictive models to assess the patients, followed by early observation and early treatment to ensure patient safety after PCNL.

There were several limitations of our study. First, this was a retrospective study from a single institute, which might lead to selection bias. Second, the patient data were not perfect; for example, the calculus load could not be calculated, and stone composition analysis could not be performed, which affects the analysis of risk factors, so we were unable to provide a more comprehensive analysis. Finally, the sample size was small and lacked representativeness. Large-scale prospective studies are needed.

\section{Conclusion}

Diabetes and operative time are independent risk factors for SIRS after PCNL, so the probability of SIRS after PCNL in patients with stones can be defined according to these two indicators, which can help clinical staff assess changes in the patient's condition and then provide early interventions to reliably prevent postoperative sepsis.

\section{Abbreviations}

PCNL:Percutaneous nephrolithotomy; 
SIRS:Systemic inflammatory response syndrome;

\section{Declarations}

Ethics approval and consent to participate: This retrospective study was approved by the hospital ethics committee (2018-237-2, No. 1 Hospital of China Medical University) 『Patients voluntarily consented to participate in the study.

Consent to publish: The authors declare that consent for publication .

Availability of data and materials: The datasets used or analysed during the current study are available from the corresponding author on reasonable request.

Competing interests: The authors declare that they have no conflict of interest.

Funding: This Paper have no Funding Sources.

Authors' contributions: JZ:study design,data collection,data interpretation,literature search and manuscript draft; MJ:literature search,data collection,statistical analysis,data interpretation; LLG:literature search,data collection; YLP:study design,literature search ,statistical analysis.

Acknowledgements: We would like to acknowledge the helpful comments on this paper received from our reviewers.

\section{References}

1. Zeng GH, Mai ZL, Xia SJ. A cross-sectional survey of urolithiasis prevalence in China Z. China J Urol. 2015; 36: 528-31.

2. Wang SG, Yu H. Percutaneous Nephrolithotomy-New Exploration for Day Operation. Journal Of Peking University. (2017; 49: 753-6.

3. Singh P, Yadav S, Singh A, Saini AK, Kumar R, Seth A, et al. Systemic Inflammatory Response Syndrome Following Percutaneous Nephrolithotomy: Assessment of Risk Factors and Their Impact on Patient Outcomes. Urol Int. 2016; 96: 207-11.

4. Ruslan K, Joseph AG, Max K, Adam CM, MantuG., Post-Percutaneous. Nephrolithotomy Systemic Inflammatory Response: A Prospective Analysis of Preoperative Urine, Renal Pelvic Urine and Stone Cultures. J Urol. 2011; 186: 189-2903.

5. Chen L, Xu QQ, Wang XF. Analysis of risk factors of systemic inflammatory response syndrome after percutaneous nephrolithotomy. Chin J Urol. 2008; 29 173-6.

6. Omar M, Noble M, Sivalingam S, El Mahdy A, Gamal A, Farag M, et al. Systemic Inflammatory Response Syndrome after Percutaneous Nephrolithotomy: A Randomized Single-Blind Clinical Trial Evaluating the Impact of Irrigation Pressure. J Urol. 2016; 196: 109-14. 
7. Zhou SL, Deng YQ, Tan F. Retrospective Analysis for the Risk Factors of Systemic Inflammatory Response Syndrome(SIRS)after Percutaneous Nephrostofithotomy(PCNL). Journal of SunYat-Sen University(Medical Science) 2016; 37: 295-9.

8. Wang QL, Liao WB, Yang SX. Retrospectively analysis of risk factors for systemic inflammatory response syndrome following retrograde intrarenal stone surgery. Int J ClinExp Med 2016; 9: 8327-33.

9. Medical Recapitulate. 2005; 11: 96.

10. Royston P. Multiple imputation of missing values The Stata Journal. 2004; 4: 227-41.

11. Kattan MW, Karpeh MS, Mazumdar M, Brennan MF. Postoperative nomogram for disease-specific survival after an R0 resection for gastric carcinoma. J Clin Oncol. 2003; 21: 3647-50.

12. Hosmer DW. Assessing the fit of the model. Applied logistic regression,. 2000: 143-202.

13. Austin PC, Steyerberg EW. Graphical assessment of internal and external calibration of logistic regression models by using loess smoothers. Stat Med. 2014; 33: 517-35.

14. Sen V, Bozkurt IH, Aydogdu O, Yonguc T, Yarimoglu S, Sen P, et al. Significance of preoperative neutrophil-lymphocyte count ratio on predicting postoperative sepsis after percutaneous nephrolithotomy. Kaohsiung J Med Sci. 2016; 32: 507-13.

15. Koras O, Bozkurt IH, Yonguc T, Degirmenci T, Arslan B, Gunlusoy B, et al. Risk factors for postoperative infectious complications following percutaneous nephrolithotomy: a prospective clinical study. Urolithiasis. 2015; 43: 55-60.

16. Yang T, Liu S, Hu J, Wang L, Jiang H. The Evaluation of Risk Factors for Postoperative Infectious Complications after Percutaneous Nephrolithotomy. Biomed Res Int. 2017; 2017: 4832051.

17. Zhu ZJ, Xu QQ, Huang XB, Hong Y, Yang QY, Wang S, et al. [Risk factor analysis of systemic inflammatory response syndrome in type 2 diabetics after percutaneous nephrolithotomy]. Beijing Da Xue Xue Bao Yi Xue Ban. 2016; 48: 643-9.

18. Wei W, Leng J, Shao H, Wang W. Diabetes, a risk factor for both infectious and major complications after percutaneous nephrolithotomy. Int J Clin Exp Med. 2015; 8: 16620-6.

\section{Figures}




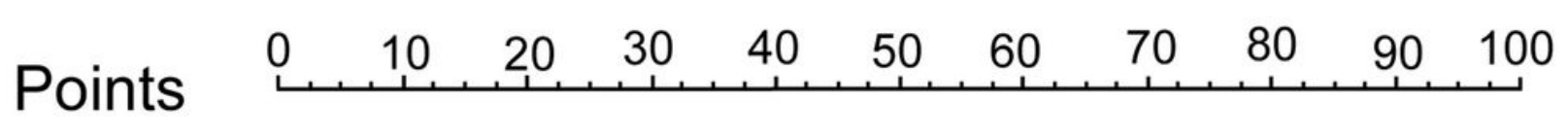

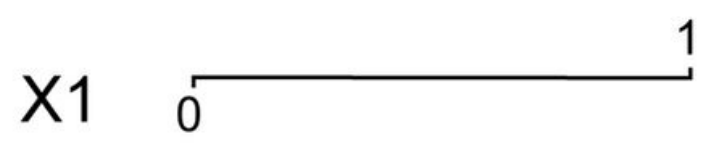

$\begin{array}{lllllllllllll}X 2 & 0 & 20 & 40 & 60 & 80 & 100 & 120 & 140 & 160 & 180 & 220 & 200\end{array}$

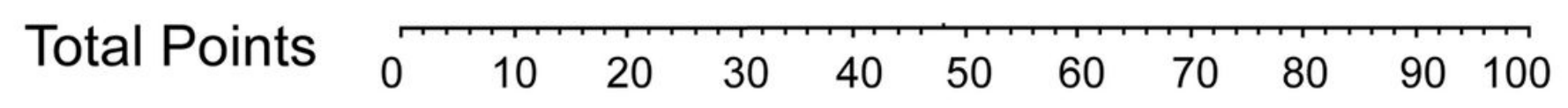

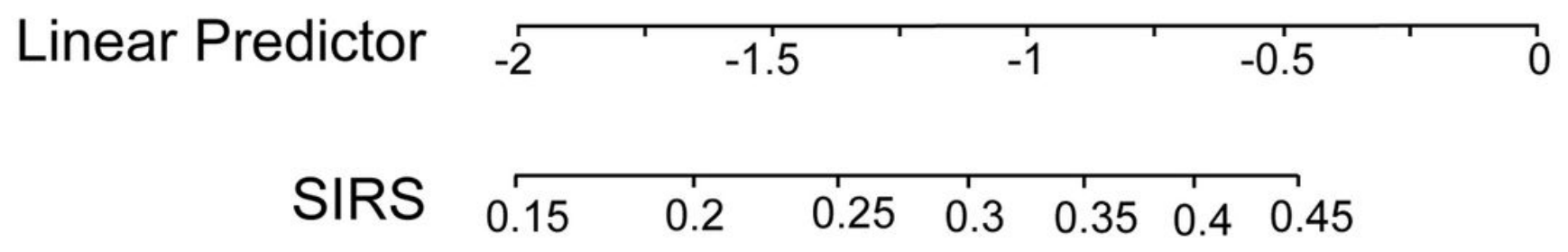

Figure 1

Nomogram to predict SIRS after PCNL. (X1=Diabetes; $\mathrm{X} 2=$ Operative time) 


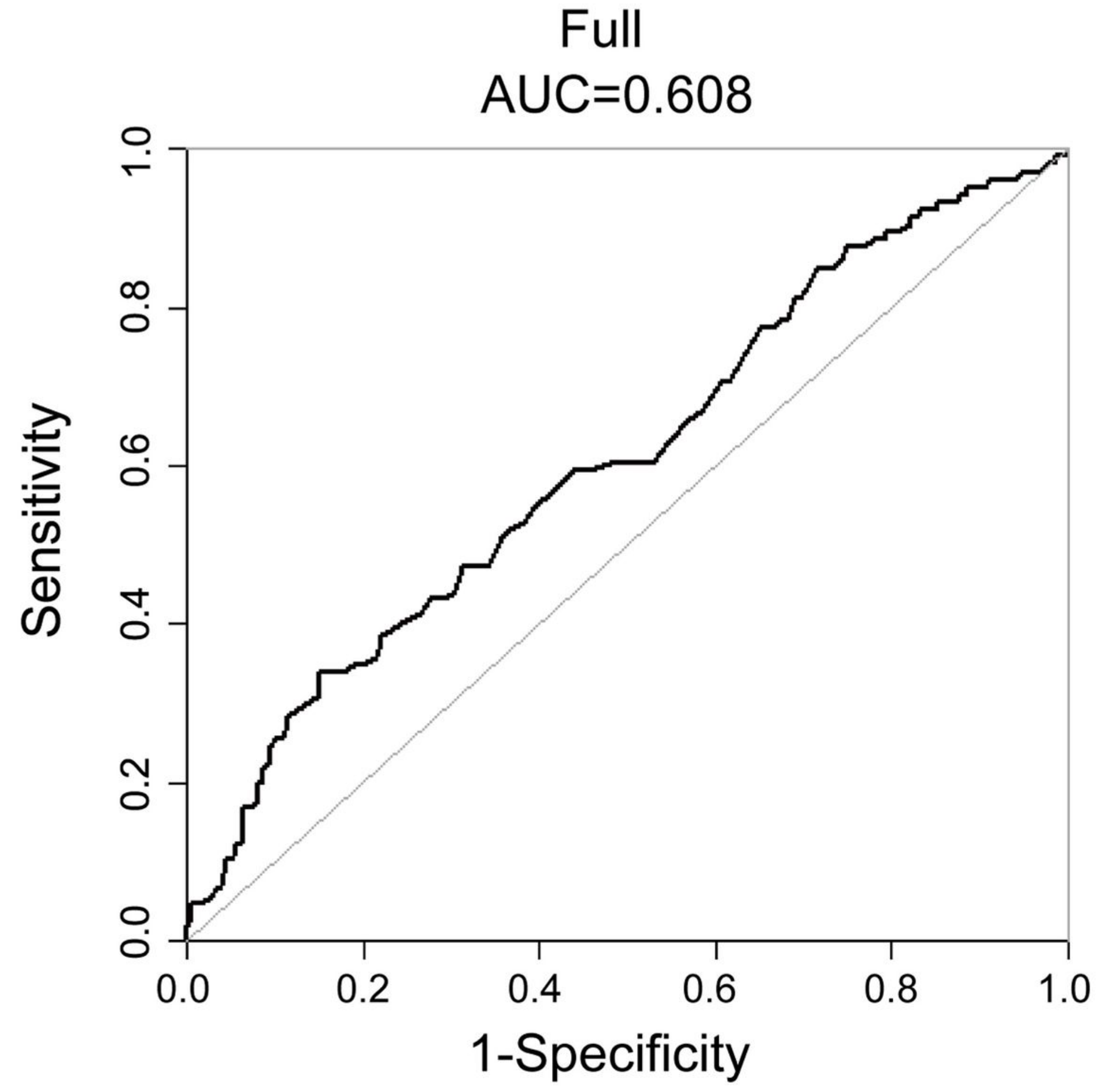

Figure 2

ROC curves for validating the discrimination power of the nomogram 


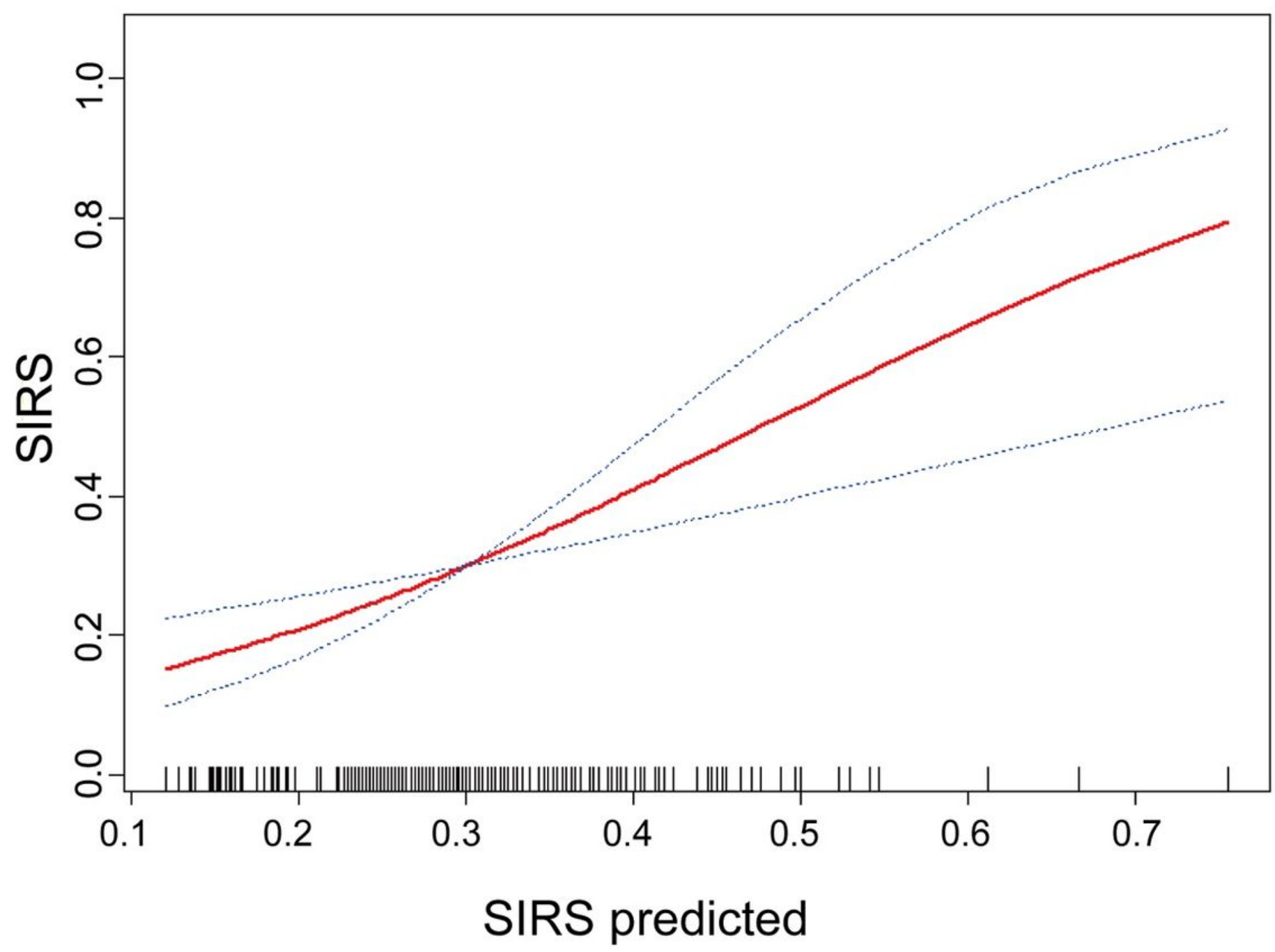

Figure 3

Calibration plots of the nomogram for the probability of SIRS after PCNL. 